\title{
Theorie eines Phantoms - Die erfolglose Suche der Prinzipientheorie nach ihrem Gegenstand
}

\author{
Ralf Poscher"
}

A. Der Prinzipienbegriff der Prinzipientheorie ............................... 349

B. Der Überflüssigkeitseinwand ......... 354

I. Die Möglichkeitsthese ............. 358

1. Rekonstruktive Lesart ........... 358

2. Konstruktive Lesart............ 361

II. Notwendigkeitsthese .............. 362
1. Das Normativitätsargument..... 363

2. Das Kollisionsargument......... 364

C. Der Existenzeinwand ................. 366

D. Warum Optimierungsgebote der Prinzipientheorie nicht ausreichen ......... 368

E. Der gute Sinn der Rede von Rechtsprinzipien ......................... 370

\section{A. Der Prinzipienbegriff der Prinzipientheorie}

In seiner Theorie der Grundrechte hat Robert Alexy die zuvor von Ronald Dworkin in dessen Auseinandersetzung mit dem angelsächsischen Positivismus stark gemachte Unterscheidung von Regeln und Prinzipien aufgegriffen und zu einer umfassenden Prinzipientheorie der Grundrechte ausgebaut. Im Kreis seiner Schüler hat sich dann eine kleine Prinzipientheorieindustrie entwickelt, die den Ansatz Alexys reformuliert ${ }^{1}$ oder für einzelne Grundrechte spezifiziert ${ }^{2}$, von den Grundrechten auf andere Rechtsgebiete übertragen ${ }^{3}$ oder auch auf die Ebene einer allgemeinen Rechtstheorie gehoben ${ }^{4}$ hat. Alexy selbst hat diese Entwicklung durch seine eigene kontinuierliche Ausweitung, Anpassung und Verfeinerung der Prinzipientheorie begleitet. Unter seiner Führung hat sich die Prinzipientheorie zu einem allgemeinen rechtstheoretischen Ansatz entwickelt, der sich aus der Einsicht in den Charakter und die Funktionsweise von Prinzipien Erkenntnisse für die unterschiedlichsten Rechtsgebiete und Rechtsfragen und für das Verständnis des Rechts im Allgemeinen verspricht. ${ }^{5}$

* Prof. Dr. Ralf Poscher lehrt an der Albert-Ludwigs-Universität Freiburg und ist einer der Direktoren des Instituts für Staatswissenschaft und Rechtsphilosophie.

1 M. Borowski, Grundrechte als Prinzipien, 2. Aufl. Baden-Baden 2007.

2 Etwa J.-R. Sieckmann, Modelle des Eigentumsschutzes, Baden-Baden 1998; M. Borowski, Die Glaubens- und Gewissensfreiheit des Grundgesetzes, Tübingen 2006.

3 Etwa J. H. Park, Rechtsfindung im Verwaltungsrecht: Grundlegung einer Prinzipientheorie des Verwaltungsrechts als Methode der Verwaltungsrechtsdogmatik, Berlin 1999; J. Pietsch, Das Schrankenregime der EU-Grundrechtecharta. Dogmatik und Bewertung auf der Grundlage einer Prinzipientheorie der Rechte, Baden-Baden 2006.

4 Die eingehendsten Ausarbeitungen bei J.-R. Sieckmann, Regelmodelle und Prinzipienmodelle des Rechtssystems, Baden-Baden 1990; ders., Recht als normatives System. Die Prinzipientheorie des Rechts, Baden-Baden 2009.

$5 \mathrm{Zu}$ den unterschiedlichen Ebenen der Prinzipientheorie R. Poscher, Einsichten, Irrtümer und Selbstmissverständnis der Prinzipientheorie, in: J.-R. Sieckmann (Hrsg.), Prinzipientheorie der Grundrechte, Baden-Baden 2007, S. 59-79; ders., Insights, Errors and Self-misconceptions of the Theory of Principles, Ratio Juris 2009, S. 425-54; ders., The Principle Theory: How Many Theories and What is Their Merit?, abrufbar unter SSRN: http://ssrn.com/abstract=1411181, demnächst in: M. Klatt (Hrsg.) Institutionalizing Reason. Perspectives on the Legal Philosophy of Robert Alexy, New York: Oxford University Press. 
Den Kern der Prinzipientheorie bildet die Unterscheidung von Regeln und Prinzipien. Für die Prinzipientheorie sind Rechtsnormen entweder Regeln oder Prinzipien. Dabei sollen sich Regeln und Prinzipien in ihrer formalen Struktur unterscheiden. Während Regeln eine Rechtsfolge definitiv anordneten, sähen Prinzipien ihre Rechtsfolgen nur prima facie vor. ${ }^{6}$ Diese unterschiedliche Struktur der beiden Normtypen manifestiere sich besonders in ihrem Kollisionsverhalten. Während im Fall einer Regelkollision entweder eine Ausnahme in die Regeln eingefügt oder eine der beiden Regeln für nichtig erklärt werden müsste, bliebe die Geltung von Prinzipien im Kollisionsfall unberührt, da sie nur Prima-facie-Wirkung beanspruchten und im Kollisionsfall erst durch optimierende Abwägung im Einzelfall zu einer definitiven Regel konkretisiert würden. ${ }^{7}$ Dem normtheoretischen Unterschied soll ein methodischer entsprechen. „Die Anwendung von Regeln erfolgt durch die Subsumtion eines Sachverhaltes unter ihren Tatbestand und Deduktion der Rechtsfolge.... Die Anwendung von Prinzipien erfordert demgegenüber... eine Abwägung kollidierender Prinzipien. “8 Die optimierungsdogmatischen Positionen der Prinzipientheorie werden dann aus einer Kombination der normtheoretischen und der methodischen Annahmen hergeleitet. Anhand des methodischen Kriteriums lässt sich eine Rechtsnorm entweder dadurch als Regel qualifizieren, dass sie durch unmittelbare Subsumtion anwendbar ist, oder sie fällt aufgrund des normontologischen Dualismus der Prinzipientheorie in die Prinzipienkategorie. Fällt eine Norm in die Prinzipienkategorie, so folgt aus der normtheoretischen Qualifikation wiederum, dass sie im Wege der optimierenden Abwägung angewandt werden muss. So sind etwa die Grundrechte keine Normen, die durch schlichte Subsumtion angewandt werden können. Aufgrund des Dualismus der Prinzipientheorie sollen sie daher Prinzipien sein. Folglich sähen sie ihre Rechtsfolgen nur prima facie vor und seien im Wege der optimierenden Abwägung anzuwenden. Grundrechte sind so bereits normtheoretisch als Optimierungsgebote ausweisbar.

Im Folgenden soll es nicht um die Leistungsfähigkeit der Prinzipientheorie in diesem oder einem anderen ihrer Anwendungsfelder gehen, ${ }^{9}$ sondern um die zentrale Prämisse der Prinzipientheorie, mit der allein sie zwar noch nicht steht, aber ohne die sie fällt: der Annahme von Prinzipien im Sinn der Prinzipientheorie.

6 R. Alexy, Theorie der Grundrechte, Frankfurt 1986, S. 88-90; Sieckmann, Regelmodelle (Fn.4), S. 67-71.

7 Alexy, Grundrechte (Fn. 6), S. 77-87; Sieckmann, Regelmodelle (Fn. 4), S. 68.

8 Sieckmann, Regelmodelle (Fn. 4), S. 18; s. auch R. Alexy, On Balancing and Subsumption, Ratio Juris 2003, S. 433.

9 Zur Kritik der Leistungsfähigkeit auf dem Gebiet der Grundrechtsdogmatik bereits R. Poscher, Grundrechte als Abwehrrechte, Tübingen 2003, S. 82-4; s. a. M. Jestaedt, Grundrechtsentfaltung im Gesetz, Tübingen 1999, S. 222-60; W. Cremer, Freiheitsgrundrechte, Tübingen 2003, S. 218-27; J. H. Klement, Vom Nutzen einer Theorie, die alles erklärt, Juristenzeitung 2008, S. 756 (760); B. Rusteberg, Der Grundrechtliche Gewährleistungsgehalt, Tübingen 2009, S. 158-166. 
Die Prinzipientheorie des Rechts setzt Rechtsprinzipien voraus. Dies scheint zunächst eine ganz unverdächtige Voraussetzung, da Juristen seit Jahrhunderten Rechtsprinzipien im Inventar ihres normativen Universums führen. Doch wenn die Prinzipientheorie von Prinzipien redet, meint sie nicht die bunte Vielfalt traditioneller Rechtsprinzipien wie den Grundsatz von Treu und Glauben, des Vertrauensschutzes, der ungerechtfertigten Bereicherung, der Gleichbehandlung etc. Die Prinzipientheorie hat vielmehr die traditionelle Rede von Rechtsprinzipien zu einem rechtstheoretischen Dualismus verschärft, nach dem das normative Universum der Juristen in exakt zwei Arten von Normen zerfällt: in unmittelbar subsumtionsfähige Regeln ${ }^{10}$ und eine bestimmte Art von Prinzipien, die in der Sphäre eines „,idealen Sollens“ angesiedelt seien und sich im realen Sollen in der Form von Optimierungsgeboten ausdrückten. Der Prinzipienbegriff der Prinzipientheorie darf also nicht mit dem traditionellen Begriff des Rechtsprinzips verwechselt werden, der weder einem normativen Dualismus noch der Idee eines „idealen Sollens“ noch dem Monismus der Optimierung verpflichtet ist. Dass nur selten wahrgenommen wird, wie anspruchsvoll die Annahme von Prinzipien im Sinn der Prinzipientheorie ist, beruht wohl wesentlich auf einer Äquivokation, in der die Prinzipien im Sinn der Prinzipientheorie mit denen unserer traditionellen Rede von Rechtsprinzipien gleichgesetzt werden. Für die Prinzipientheorie reicht es aber gerade nicht aus, auf traditionelle Rechtsprinzipien zu verweisen, sondern sie muss zeigen, dass Prinzipien in ihrem besonderen Verständnis des Begriffs eine eigene normative Entität bilden, die sich strukturell, und nicht lediglich hinsichtlich ihres mehr oder weniger abstrakten Inhalts oder ihrer mehr oder weniger großen Bedeutung, von anderen Normen unterscheidet. Gerade aber auch innerhalb der Prinzipientheorie ist höchst umstritten, was Prinzipien im Sinn der Prinzipientheorie sein sollen, wie sie sich beschreiben und theoretisch rekonstruieren lassen. Es ist jedenfalls bereits in der ersten Annäherung an die Prinzipientheorie eine Auffälligkeit, dass zwischen ihren Anhängern keine Einigkeit darüber besteht, was den zentralen Gegenstand ihrer Theorie bilden soll. ${ }^{11}$

Zunächst hatte Robert Alexy Prinzipien mit Optimierungsgeboten identifiziert. In seiner Arbeit zur Grundlegung der Prinzipientheorie als „Theorie der Grundrechte“ heißt es in dem zentralen Abschnitt „Prinzipien als Optimierungsgebote“ noch: „Der für die Unterscheidung von Regeln und Prinzipien entscheidende Punkt ist, daß Prinzipien Normen sind, die gebieten, daß etwas in einem relativ auf die rechtlichen und tatsächlichen Möglichkeiten möglichst hohen Maß realisiert wird. Prinzipien sind demnach Optimierungsgebote... Demgegenüber sind Regeln Normen, die stets

10 Zur Kritik des Regelbegriffs der Prinzipientheorie Poscher, Einsichten (Fn. 5), S. 70-73; ders. Insights (Fn. 5), S. 438-41.

11 S. etwa die erneute Kritik an Alexys Prinzipienbegriff bei Sieckmann, Normatives System (Fn. 4), S. $22 \mathrm{f}$. 
nur entweder erfüllt oder nicht erfüllt werden können."12 Im Ausgangspunkt der Prinzipientheorie waren Prinzipien schlicht Optimierungsgebote. Darin lag gerade auch die Pointe des Prinzipienkonzepts Alexys, der den Prinzipienbegriff Dworkins dadurch präzisierte, dass er die Rede vom Prima-facie-Sollen als Optimierungsgedanken rekonstruierte. ${ }^{13}$ Optimierung in diesem Sinn bedeutet die Relativierung einer Rechtsfolge einer Norm auf die tatsächlichen und rechtlichen Möglichkeiten, ${ }^{14}$ die wiederum die von Dworkin angesprochene Dimension des Gewichts präzisiert. In der präzisierenden Rekonstruktion der Prinzipien Dworkins als Optimierungsgebote lag die analytische Leistung der frühen Prinzipientheorie.

Schon bald wurde aber von den Anhängern der Prinzipientheorie selbst gesehen, dass Optimierungsgebote alle Merkmale einer Regel im Sinn der Prinzipientheorie aufweisen. Wie jedes andere Gebot können sie strikt und vollständig erfüllt werden, indem die geforderte Optimierung vorgenommen wird. Verpflichtet eine Norm die Halter von Kraftfahrzeugen den Reifendruck im Hinblick auf das Ladegewicht, den Kraftstoffverbrauch und die Sicherheit zu optimieren, sind die Kraftfahrzeughalter zu eben dieser Optimierung strikt verpflichtet. Jan Sieckmann, der Schüler Alexys, der auf den Regelcharakter von Optimierungsgeboten aufmerksam machte, stellte daher fest: „Damit bleibt jedoch das Problem, daß Optimierungsgebote ... gerade die für Regeln i.e.S. charakteristischen Merkmale der strikten Geltung bzw. definitiven Erfüllbarkeit aufweisen. "15 Ein Problem ist die Erkenntnis, dass sich Optimierungsgebote strukturell nicht von anderen Normen unterscheiden, freilich nur für die Prinzipientheorie. Auf der Grundlage eines traditionellen Prinzipienverständnisses, das zwischen Rechtsprinzipien und anderen Rechtsnormen lediglich graduelle Unterschiede sieht, würde die Einsicht nicht überraschen. Sie würde die traditionelle Sicht lediglich bestätigen, in dem sie zeigt, dass das traditionelle Prinzipienverständnis auch auf Rechtsprinzipien zutrifft, die als Optimierungsgebote rekonstruiert werden.

Die Prinzipientheorie brachte der Wegfall der Optimierungsgebote als Kandidaten für den Prinzipienbegriff jedoch in eine prekäre Lage: Sie drohte ihren Gegenstand zu verlieren. Es wundert daher nicht, dass die Vertreter der Prinzipientheorie große Anstrengungen unternommen haben, doch noch mit Prinzipien aufwarten zu können, die ihren rechtstheoretischen Dualismus bestätigen könnten. So war für Robert Alexy die Entdeckung, dass Optimierungsgebote keine Prinzipien sind, kein Anlass zur Revision der Prinzipientheorie, sondern der fehlende Prinzipiencharakter der

12 Alexy, Grundrechte (Fn. 6), S. 75 f. - Hervorhebung im Original.

13 Alexy, ebd., S. 87-90.

14 Alexy, ebd., S. 75.

15 Sieckmann, Regelmodelle (Fn. 4), S. 65. 
Optimierungsgebote „wirft auf sie nur ein schärferes Licht. “16 Seither vertritt Alexy, dass Prinzipien ein „ideales Sollen“ darstellen. ${ }^{17}$

Gegenüber entsprechenden Rettungsversuchen liegt der Einwand nahe, dass sie eben nur das sind, dass die Annahme eines „idealen Sollens“ einzig dem Zweck diene, den der Prinzipientheorie mit den Optimierungsgeboten abhandengekommenen Gegenstand zu fingieren, ohne dass das „,ideale Sollen“ für die Erklärung von normativen Phänomenen wie den Optimierungsgeboten eine Erklärungsleistung erbringt. ${ }^{18}$ Alexy hat nun auf diese Kritik reagiert und seine Konzeption der Prinzipien als „,ideales Sollen" verteidigt. ${ }^{19} \mathrm{Er}$ hat darauf hingewiesen, dass die Kritik zwei Einwände formuliere: zum einen den ontologischen, dass keine Prinzipien im Sinn der Prinzipientheorie existieren, dass es neben dem realen, kein ideales Sollen gibt; zum anderen den Einwand der Überflüssigkeit, nach dem, selbst wenn sich ein „ideales Sollen“ konstruieren ließe, es jedenfalls nicht benötigt würde, um Phänomene der Rechtsordnung zu erklären, denen die Prinzipientheorie gilt. „Der ontologische Einwand ist der radikalere. Wenn er zuträfe, träfe auch der Überflüssigkeitseinwand zu. “20

Die folgende Auseinandersetzung mit Alexys Replik widmet sich nicht nur aus dramaturgischen Gründen zunächst seiner Entgegnung auf den Überflüssigkeitseinwand - die Überflüssigkeit folgt sonst bereits aus der Nicht-Existenz. Für dieses Vorgehen spricht auch ein strategischer Grund. Während sich die Vertreter der Prinzipientheorie weitgehend einig darüber sind, wozu sie der Annahme von Prinzipien zu bedürfen glauben - nämlich zur Erklärung der Funktionsweise von Optimierungsgeboten und ihrer normativen Eigenschaften -, vertritt fast jeder Prinzipientheoretiker eine eigene These dazu, was Prinzipien neben Optimierungsgeboten sein sollen. ${ }^{21}$ Das Angebot spannt sich mittlerweile über fast ein halbes Dutzend Vorschläge. Die Entwicklung der Prinzipientheorie nährt den Verdacht, dass es die Kritik mit einem hydra-artigen Phänomen zu tun hat. Für jeden Kandidaten des Prinzipienbegriffs, den die Kritik zurückweist, sprießen gleich mehrere neue hervor. ${ }^{22}$ Nicht nur wegen der allgemeinen Probleme negativer Existenzbeweise, sondern auch wegen der Promiskuität des Prinzipienbegriffs der Prinzipientheorie wird die Überflüssigkeitsthese dem vielstimmigen Chor der Prinzipientheoretiker daher besser gerecht. So wie ein Verteidiger

16 R. Alexy, Zur Struktur von Rechtsprinzipien, in: B. Schilcher/P. Koller/B.-C. Funk (Hrsg.), Regeln, Prinzipien und Elemente im System des Rechts, Wien 2000, S. 31 (38f.).

17 Alexy, ebd.

18 Poscher, Einsichten (Fn. 5), S. 10-12; ders., Insights (Fn. 5), S. 436-38; ders., Principles (Fn. 5), S. 25-28.

19 R. Alexy, Ideales Sollen, in: L. Clérico/J.-R. Sieckmann (Hrsg.), Grundrechte, Prinzipien und Argumentation, Baden-Baden 2009, S. 21-22.

20 Ebd.

21 Einen Überblick bietet etwa Borowski, Prinzipien (Fn. 1), S. 68-105.

22 Ein neuer Spross nun bei M. Reßing, Prinzipien als Normen mit zwei Geltungsebenen, ARSP 95 (2009), S. 28-48. 
der Gesetze der Mechanik die Existenz von Engeln offen lassen kann, wenn er ihrer zur Erklärung physikalischer Phänomene nicht mehr bedarf, kann ein Kritiker der Prinzipientheorie die Existenz von Prinzipien als „,idealem Sollen “23 oder „pro tanto Sollen“ 24 oder „unendlich iterierten Geltungsgeboten“ 25 oder „Normen mit zwei Geltungsebenen " 26 offen lassen, wenn gezeigt werden kann, dass es neben der Annahme von Optimierungsgeboten, die nach der dualistischen Ontologie der Prinzipientheorie gerade keine Prinzipien sind, keiner weiteren normativen Entitäten bedarf, um die normativen Phänomene zu erklären, um die es der Prinzipientheorie geht. Wenn sich die Überflüssigkeitsthese bewährt, wird die Existenzthese uninteressant, mag sie auch im Dutzend angeboten werden. Die Prinzipientheoretiker mögen sich dann gleichsam darüber streiten, ob Engel körperliche Wesen sind, Flügel haben und weiße Nachthemden tragen, folgen würde daraus nichts.

Zunächst soll der Überflüssigkeitseinwand erläutert und gegenüber der Replik von Alexy erhärtet werden (B.). Auf den Existenzeinwand wird nur hinsichtlich des „idealen Sollens“ eingegangen, das Alexy selbst mit dem Prinzipienbegriff identifiziert (C.). In einem dritten Abschnitt soll erklärt werden, warum die Vertreter der Prinzipientheorie sich nicht einfach damit begnügen können, Optimierungsgebote zu untersuchen (D.). Abschließend wird noch einmal klargestellt, dass sich die Kritik der Prinzipientheorie nicht gegen die Annahme von Rechtsprinzipien im traditionellen Sinn richtet und in welchem Sinn die Rede von Rechtsprinzipien jenseits aller theoretischen Aufladung durch die Prinzipientheorie ihren guten Sinn behält (E.).

\section{B. Der Überflüssigkeitseinwand}

Wenn Optimierungsgebote keine Prinzipien im Sinne der Prinzipientheorie sind, dann müssen Prinzipien anderswo zu finden sein. Nun würde es der Prinzipientheorie nicht weiterhelfen, den Prinzipienbegriff völlig von den Optimierungsgeboten zu lösen. Die Pointe der dogmatischen Vorschläge der Prinzipientheoretiker liegt ja gerade darin, alle möglichen Normen aufgrund ihrer Prinzipieneigenschaft als Optimierungsgebote zu verstehen und alle Rechtskonflikte durch optimierende Abwägung zu lösen. Sowenig wie die normstrukturelle Unterscheidung von Regeln und Prinzipien aufgeben werden darf, sowenig dürfen Prinzipien mit etwas anderem als den Optimierungsgeboten in Verbindung gebracht werden. Würden Prinzipien mit etwas anderem als Optimierungsgeboten in Verbindung gebracht, ließe sich aus dem rechtstheoretischen Dualismus nicht mehr auf eine Abwägungsdogmatik anhand des

23 Alexy, Strukur (Fn. 16), S. 31 (38 f.); ders., Ideales Sollen (Fn. 19).

24 N. Jansen, Die Struktur der Gerechtigkeit, 1998, S. 101.

25 J.-R. Sieckmann, Zur Analyse von Normkonflikten und Normabwägungen, in: G. Meggle (Hrsg.), Analyomen, 2. Proceedings of the 2nd Conference "Perspectives in Analytical Philosophy", Bd. III, 1994, S. 349-52.

26 Reßing, Prinzipien (Fn. 22), ebd. 
Optimierungsgedankens - etwa für die Grundrechte - schließen. So überrascht es nicht, dass Alexy die Prinzipien im Sinne der Prinzipientheorie innerhalb der Optimierungsgebote findet. Optimierungsgebote seien zwar keine Prinzipien, aber in den Optimierungsgeboten seien Prinzipien am Werk. Für Alexy ist „zwischen den $z u$ optimierenden Geboten und Geboten zu optimieren zu unterscheiden. Die zu optimierenden Gebote sind die Abwägungsgegenstände. Man kann sie als ,ideales Sollen‘ oder ,Ideale“ bezeichnen. "27 Prinzipien sollen nun nicht die Optimierungsgebote als solche sein, sondern das „,ideale Sollen“, das den Gegenstand der Optimierung bilde. Optimierungsgebote sind danach eine besondere Art von Regeln, die gleichsam ein Prinzip inkorporieren. Auch wenn Optimierungsgebote im Bereich des realen Sollens nur profanen Regelcharakter haben, stehen sie durch ihren besonderen Gegenstand in Verbindung mit dem „idealen Sollen“, den Prinzipien.

Die Kritik gegen diese Rekonstruktion der Prinzipien als Gegenstand der Optimierungsgebote geht dahin, dass sie den Gegenstand der Optimierungsgebote missversteht. Nach der von Alexy vorgeschlagenen Rekonstruktion ist der Gegenstand des Optimierungsgebots seinerseits ein Gebot, ein Gebot mit idealem Charakter, ein „,ideales Sollen“. Der Rekonstruktionsversuch verkennt jedoch, dass der Gegenstand von Optimierungsgeboten regelmäßig kein Gebot, keine Norm ist, sondern ein empirischer Sachverhalt. Optimierungsgebote geben regelmäßig nicht auf, Normen zu optimieren, sondern richten sich auf faktische Gegenstände. Optimierungsgebote sind in der Regel nicht reflexiv in dem Sinn, dass sie Gebote zur Optimierung von Geboten - geschweige denn von idealen Geboten - zum Ausdruck bringen. Ein auf die Meinungsäußerungsfreiheit bezogenes Optimierungsgebot gibt auf, den empirischen Sachverhalt der Freiheit der Meinungsäußerung zu optimieren; es gibt nicht auf, das Gebot der Freiheit der Meinungsäußerung zu optimieren. Optimierungsgeboten geht es in der Regel um die Verbesserung empirischer Sachverhalte, nicht reflexiv um die Verbesserung anderer Normen. Sie fordern Meinungsfreiheit, nicht eine Verbesserung der Gebote der Meinungsfreiheit. Entsprechend kommt ein repressiver Staat dem Gebot, die Meinungsfreiheit zu optimieren, auch nicht dadurch nach, dass er noch eine internationale Konvention zum Schutz der Meinungsfreiheit unterzeichnet - also gleichsam das Gebot, durch eine internationale Verpflichtung optimiert -, sondern nur dadurch, dass er die Zensur tatsächlich abschafft.

Entgegen Vertretern der Prinzipientheorie müssen die Gegenstände von Optimierungsgeboten auch keinen normativen Charakter haben, um die Normativität von Optimierungsgeboten zu erklären. ${ }^{28}$ Normativität erhalten die Gegenstände der Optimierungsgebote durch das in den Optimierungsgeboten enthaltene Gebot, einen Gegenstand relativ zu den rechtlichen und faktischen Möglichkeiten zu steigern. Die

27 Alexy, Struktur (Fn. 16), S. 31 (38 f.); ders., Ideales Sollen (Fn. 19).

28 So aber Sieckmann, Regelmodelle (Fn. 4), S. 66. 
Normativität der Optimierungsgebote ergibt sich allein aus dem Gebot zu optimieren, eines $z u$ optimierenden Gebots als Gegenstand der Optimierung bedarf es dafür nicht. Optimieren lassen sich beliebige Gegenstände: Reifendruck, Gesundheit, Meinungsfreiheit; auf Normen als Optimierungsgegenstände sind Optimierungsgebote nicht angewiesen. Dabei wird meist durch den Kontext des Gebots festlegt, ob der Gegenstand relativ zu den rechtlichen oder faktischen Möglichkeiten maximiert oder minimiert werden soll. Als Optimierungsgebot verstanden, verlangt etwa das Grundrecht auf körperliche Unversehrtheit, dass die Eingriffe in die Gesundheit relativ zu den rechtlichen und faktischen Möglichkeiten minimiert und nicht maximiert werden. Anders wäre in der Arbeitslosenverwaltung ein Gebot zur Optimierung der Beschäftigtenzahlen zu verstehen.

Dass Optimierungsgebote zwar selbst Gebote sind, aber in der Regel nicht wieder auf ein Gebot bezogen werden, wird auch im Vergleich mit Fällen deutlich, in denen dies ausnahmsweise einmal anders ist. Optimierungsgebote können sich auf jeden Gegenstand beziehen. Sie können daher auch normative Gegenstände haben. Auch Normen lassen sich optimieren und damit auch Gebote. So können etwa Regelungen des Steuerrechts, die Steuerschlupflöcher lassen, durch den Erlass von Gesetzen optimiert werden, die diese Lücken schließen. Doch bilden entsprechende Optimierungsgebote in der Rechtsordnung eher die Ausnahme als die Regel. So sind die Grundrechte, die der Prinzipientheorie als Paradigma für Optimierungsgebote gelten, auch wenn sie als Optimierungsgebote verstanden werden, regelmäßig nicht auf die Optimierung von Geboten, ${ }^{29}$ sondern auf die Optimierung empirischer Sachverhalte gerichtet.

Gegenüber dieser Kritik räumt Alexy nun ein: „Auf den ersten Blick scheint dieser Einwand berechtigt zu sein, denn es besteht die Möglichkeit, den Gegenstand eines Gebots... unmittelbar zum Gegenstand einer Optimierung zu machen. Aus

(2) $\mathrm{Op}$

würde dann

(4) OOptp

29 Etwas anderes könnte für normgeprägte Grundrechte gelten. 
werden. " ${ }^{30}$ In diesem Zitat ist $O p$ in (2) als Abkürzung für die mit unterschiedlichen Sätzen ausdrückbare Proposition zu lesen, dass es geboten ist, dass $p .{ }^{31}$ In (4) soll OOpt $p$ für die Proposition stehen, dass es geboten ist, den Gegenstand von $p z u$ optimieren.

So erfreulich das Zugeständnis der initialen Plausibilität des Überflüssigkeitseinwands auch ist, muss aber bereits gegenüber dieser Konzession darauf hingewiesen werden, dass sie in (4) eine irreführende Notation verwendet. In (2) steht $p$ - den Standardnotationen entsprechend - als Variable für eine Proposition. In dem Beispiel, das Alexy an dieser Stelle gibt, steht $p$ etwa für die Proposition, dass Notleidenden geholfen wird. Op ist daher, auf dieses Beispiel bezogen, zu lesen als: Es ist geboten, dass Notleidenden geholfen wird. In (4) steht der Ausdruck Opt hingegen an der Stelle eines Operators, obwohl Opt lediglich die Proposition $p$ qualifiziert. ${ }^{32}$ In dem Beispiel steht Opt $p$ dafür, dass die Hilfe für Notleidende optimiert wird. Opt qualifiziert das, was geboten ist - im Beispiel ist nicht schlicht die Hilfe für Notleidende geboten, sondern die Optimierung der Hilfe für Notleidende. Durch die Qualifikation wird zum Ausdruck gebracht, dass sich die Proposition Opt $p$ derart von $p$ unterscheidet, dass sie die Optimierung des Gegenstands von $p$ zum Gegenstand hat. Klarer ließe sich dies etwa indexikalisch als $p_{\text {Opt }}$ ausdrücken. Unabhängig von der Idiosynkrasie der von Alexy verwendeten Notation bleibt festzuhalten, dass Opt eine Qualifikation der Proposition $p$ und keinen Operator markiert. Mit der Freiheit der Notation verbindet sich keine Freiheit in der Sache.

Um den auch ihm plausiblen geltenden Einwand zu entkräften, geht Alexy in seiner Replik zweistufig vor. Erstens will er zeigen, dass die profane Rekonstruktion von Optimierungsgeboten nicht die einzig mögliche ist, sondern auch eine Rekonstruktion anhand eines „idealen Sollens“ eingeführt werden kann. Zweitens will er zeigen, dass die Rekonstruktion anhand eines „,idealen Sollens“ nicht nur möglich, sondern auch notwendig ist, um bestimmte Eigenschaften von Optimierungsgeboten zu er-

30 Alexy, Ideales Sollen (Fn. 19), S. 24.

31 Die Formalisierung entspricht der Schreibweise der deontischen Standardlogik. Diese geht zurück auf von Wright, der den Ausdruck $O A$ als Abkürzung für die Proposition, dass die mit A bezeichnete Handlung geboten ist, einführte (G. H. von Wright, Deontic Logic, Mind 60 (1951), S. 4). Das Zeichen $O$ wurde dabei in Anlehnung an das englische Wort „obligatory“ ausgewählt. Aus von Wrights initialem Entwurf einer (Handlungs-)Logik, in welcher der Operator $O$ ausschließlich auf Handlungen angewandt wurde, entwickelte sich durch eine Vielzahl von Arbeiten verschiedener Autoren die deontische Standardlogik, in der analog zur Prädikatenlogik Variablen und Operatoren propositional interpretiert werden (vgl. etwa A. N. Prior, Formal Logic, Oxford: Oxford University Press 1962 und A. R. Anderson, The Formal Analysis of Normative Systems, in: N. Rescher (Hrsg.), The Logic of Decision and Action, Pittsburgh: University of Pittsburgh Press, 1956). Später übernahm von Wright selbst diese Auffassung (vgl. z.B. G. H. von Wright, A New System of Deontic Logic, Logical Studies, London: Routledge and Keagan Paul Ltd, 1957, S. 58-75; ders., An Essay in Deontic Logic and the General Theory of Action, Amsterdam: North Holland Publishing Company 1968). Dementsprechend ist - gemäß den Konventionen der deontischen Standardlogik - der Operator $O$ als Abkürzung für die Proposition, dass es geboten ist, und $p$ als Abkürzung für eine beliebige Proposition, dass $p$, zu lesen.

32 Opt lässt sich auch nicht als Prädikat einer Individuenvariabel $p$ verstehen. Dies verbietet bereits das Gebot der Notationskonstanz, da $p$ in (2) eine Proposition und keine Individuenvariabel notiert. 
klären. Demgegenüber soll gezeigt werden, dass Optimierungsgebote weder durch normativ aufgeladene Optimierungsgegenstände zutreffend rekonstruiert werden können, noch entsprechende Konstruktionen für die Erklärung der Eigenschaften von Optimierungsgeboten erforderlich sind.

\section{Die Möglichkeitsthese}

Neben der profanen Art Optimierungsgebote als

OOptp

zu rekonstruieren, gibt es für Alexy „auch die Möglichkeit, das Optimierungsgebot so zu konstruieren, daß der Optimierungsgegenstand einen normativen Charakter erhält... Das Optimierungsgebot nimmt dann die folgende Form an:

(5) OOpt Op. “33

Die Möglichkeitsthese Alexys ist ambivalent. Zum einen lässt sie sich rekonstruktiv verstehen. Als rekonstruktive These besagte sie, dass sich ein beliebiges Optimierungsgebot (OOpt $p$ ) als Gebot zur Optimierung eines Gebots (OOpt Op) rekonstruieren lässt. Als konstruktive These besagte sie, dass sich unabhängig von Optimierungsgeboten, die sich unmittelbar auf einen faktischen Optimierungsgegenstand beziehen, auch Optimierungsgebote konstruieren lassen, die in einem reflexiven Sinn ein Gebot zum Optimierungsgegenstand haben. Als rekonstruktive These ist die Möglichkeitsthese Alexys jedoch schlicht falsch; als konstruktive These ist sie trivial und für die Optimierungsgebote, denen die Prinzipientheorie gilt, uninteressant.

\section{Rekonstruktive Lesart}

In den Passagen, die vom „idealen Sollen“ handeln, bezieht sich Alexy ausschließlich auf Optimierungsgebote, die faktische Optimierungsgegenstände aufweisen, wie etwa die Hilfe für Notleidende. Bei keinem seiner konkreten Beispiele für Optimierungsgebote geht es um Normen, die aufgeben, andere Normen zu optimieren. Wenn er nun von der Möglichkeit spricht, die Optimierungsgebote, die er exemplarisch anführt, als Gebote zur Optimierung eines normativen Gegenstands zu rekonstruieren, geht er scheinbar davon aus, dass ein einem empirischen Sachverhalt geltendes Optimierungsgebot als Gebot zur Optimierung eines normativen Gegenstands rekonstruiert werden kann. Dafür spricht besonders auch, dass für ihn dem Gebot, Notleidenden zu helfen, als ideales Sollen verstanden, das Optimierungsgebot

OOpt Op 
entsprechen soll. ${ }^{34}$ Doch auch wenn es sich bei $p$ um denselben propositionalen Gehalt handelt, entspricht die von Alexy vorgeschlagene Rekonstruktion nicht dem Optimierungsgebot, das er zu rekonstruieren meint:

OOpt $\mathrm{Op} \neq \mathrm{OOpt} p$.

Dies zeigt sich, wenn man für $p$ eine beliebige Proposition einsetzt. Das Gebot, die Gesundheit zu optimieren (OOpt p), ist ein anderes Optimierungsgebot als das Gebot, das Gebot der Gesundheit zu optimieren (OOpt $O p$ ). Die Gesundheit kann man etwa durch medizinische Behandlungen oder Fitnessprogramme optimieren; das Gebot, die Gesundheit zu erhalten oder wiederherzustellen, lässt sich hingegen vielleicht dadurch optimieren, dass man an gesundheitsschädliche Verhalten Sanktionen wie etwa erhöhte Krankenversicherungsprämien oder eine schlechtere medizinische Versorgung knüpft - durch kneippsche Anwendungen lässt sich die Effektivität des Gebots, gesund zu leben, jedoch nicht beeinflussen. Die Optimierung eines Gebots ist etwas grundsätzlich anderes als die Optimierung eines faktischen Sachverhalts. Wird dies übersehen, führt dies zu Kategorienfehlern.

Dies gilt auch für Alexys eigenes Beispiel. Alexy geht von dem Gebot, Notleidenden zu helfen $(p)$ aus. Alexy räumt zunächst ein, dass es möglich ist, den Gegenstand des Gebots „die Hilfe für Notleidende ( $p$ ), unmittelbar zum Gegenstand der Optimierung zu machen. Aus

(2) $\mathrm{Op}$

würde dann

(4) OOptp

werden.“ 35 Es sei jedoch auch möglich, dass man „das Gebot, Notleidenden zu helfen, also (2), zum Gegenstand der Optimierung macht. Das Optimierungsgebot nimmt dann die folgende Form an:

(5) OOpt Op. “36

Das einfache Gebot, die Hilfe für Notleidende zu optimieren (OOpt p), hat aber einen anderen Inhalt als das reflexive Gebot, das Gebot, Notleidenden zu helfen, zu optimieren $(O O p t O p)$. Die Hilfe für Notleidende lässt sich optimieren, indem man Zelte und Nahrungsmittel verteilt. Das Gebot, Notleidenden zu helfen, lässt sich hingegen allenfalls dadurch optimieren, dass man das Strafmaß für den Tatbestand der unterlassenen Hilfeleistung erhöht oder seinen Anwendungsbereich über Unglücksfälle hinaus erstreckt. Das einfache Gebot ist ein anderes als das reflexive.

34 Ebd. S. 25.

35 Ebd.

36 Ebd. 
Dies zeigt sich ferner an den jeweils unterschiedlichen rechtlichen und faktischen Möglichkeiten, die für die Optimierung der beiden Gebotsgegenstände von Bedeutung sind. Die Hilfe für Notleidende findet ihre rechtlichen Grenzen u.U. in den Eigentumsrechten Dritter und ihre faktischen in den zur Verfügung stehenden materiellen Ressourcen. Die rechtlichen und faktischen Grenzen der Optimierung des Gebots zur Hilfeleistung sind hingegen ganz anderer Art: rechtlich finden sie sich u.U. in dem Erfordernis der Verhältnismäßigkeit von Sanktionen für Gebotsverstöße, faktisch in den begrenzten staatlichen Überwachungsressourcen. Als rekonstruktive ist die Möglichkeitsthese Alexys so offensichtlich falsch, dass trotz gegenläufiger Indizien im Text, der Grundsatz der wohlwollenden Interpretation dagegen spricht, ihm die rekonstruktive Lesart zu unterstellen.

In der Sache nicht weiter führt auch ein Notationsmanöver, mit dem Alexy das „ideale Sollen“ auf den deontischen Operator zu verschieben versucht. Er will nun seine frühere These aufgeben, dass allein die normative Qualität des Optimierungsgegenstands bereits Prinzipien und ein „ideales Sollen“ hervorbringt. „Damit ziehe ich die... These, dass das ideale und das reale Sollen eine Frage des Gebotsgegenstands sei und die ontische Modalität als solche nicht betreffe, zurück. “ ${ }^{37}$ Nun sei vielmehr der entscheidende Punkt, „daß sich nicht nur der Gebotsgegenstand qualifizieren lässt. Auch die Modalitäten des Gebots selbst sind einer Qualifikation zugänglich. Besteht die Qualifikation in einer Idealisierung, so führt dies zum idealen Sollen. Die Idealisierung der Modalität des Gebots lässt sich durch , $\mathrm{O}_{\mathrm{i}}{ }^{\prime}$ ausdrücken. ,Idealisierung' bedeutet dabei, dass von entgegenstehenden Rechten und Pflichten abstrahiert wird. “38

$\mathrm{O}_{i} p$

soll dann der Ausdruck des „idealen Sollens“ sein und das gegenseitige Implikationsverhältnis

$$
\text { „OOpt Op } \leftrightarrow \mathrm{O}_{i} p \text { “39 }
$$

gelten. Indes ist $O_{\mathrm{i}}$ weder ein normativer Operator, der ein ideales Sollen zum Ausdruck bringt, noch entspricht $O_{\mathrm{i}} p$, wie es von Alexy definiert wird, OOpt $\mathrm{O} p$; noch beträfe ein $O_{i} p$, das $O O p t O p$ entspricht, den Optimierungsgeboten, um die es der Prinzipientheorie zu tun ist.

Ein Gebot, das unabhängig von rechtlichen und faktischen Möglichkeiten $p$ gebietet, hat die Form $O p$, eines Indexes bedarf es dafür nicht. Ein von den entgegenstehenden rechtlichen Pflichten abstrahierendes Gebot von $p$ ist gerade nicht äquivalent mit 
OOpt Op: zum einen nicht, weil OOpt Op mit Opt $\mathrm{Op}$ einen anderen Gegenstand hat; zum anderen nicht, weil die Optimierung $(O p t)$ gerade die Relativierung auf die rechtlichen und faktischen Möglichkeiten ausdrückt. Wenn $O O p t O p$ und $O_{i}$ äquivalent sein sollen, dann wird lediglich $\mathrm{Opt} O \mathrm{zu}$ einem Index $i$ zusammengezogen. Der Ausdruck Opt $\mathrm{O}$ ist jedoch Teil der Proposition Opt Op. Gegenstand des Gebots $\mathrm{OO} p t \mathrm{O} p$ ist nicht $p$, sondern die Optimierung des Gebots von $p$ (Opt Op). Der Index $i$ ist nur eine andere Schreibweise für einen Teil der Proposition Opt Op. So wie Opt eine Qualifikation der Proposition $p$ zum Ausdruck bringt, ${ }^{40}$ so qualifiziert auch Opt $\mathrm{O}$ den Gegenstand der Proposition $p$. Opt Op bringt die Proposition zum Ausdruck, dass das Gebot des Gegenstands der Proposition $p$ optimiert wird. Opt $\mathrm{O}$ ist kein Index des normativen Operators $\mathrm{O}$, wie Alexy mit der Notation $\mathrm{O}_{\mathrm{i}}$ suggeriert, sondern der Proposition $p$, die - wenn $i$ für Opt $O$ stehen soll - als $p_{\mathrm{i}} \mathrm{zu}$ notieren wäre. Wie aus dem neuen und erneut irreführenden Notationsvorschlag Alexys ein „ideales Sollen“ entstehen soll, das von OOpt Op verschieden sei, das nun auch nach Alexy noch kein Prinzip oder „,ideales Sollen“ zum Gegenstand hat, bleibt unerfindlich. Hinzu kommt, dass sich das ganze Notationsmanöver auf das reflexive Gebot OOpt Op bezieht, das - wie gezeigt - nicht die Optimierungsgebote betrifft, um die es der Prinzipientheorie geht und von denen Alexy einzig handelt.

\section{Konstruktive Lesart}

Ist die rekonstruktive Lesart um Alexys Möglichkeitsthese offensichtlich falsch, trifft für die konstruktive Lesart das Gegenteil zu: Sie ist so offensichtlich richtig, aber auch so trivial, dass aus ihr nichts für die Fragen folgt, die die Prinzipientheorie zum Gegenstand hat. Wenn mit der Möglichkeitsthese lediglich gemeint wäre, dass sich unabhängig von den Geboten, die Alexy diskutiert, wie etwa dem Gebot, Notleidenden zu helfen, auch noch ganz andere Gebote konstruieren lassen, die nicht die Hilfe für Notleidende, sondern Fragen der Optimierung etwa des strafrechtlichen Tatbestands der unterlassenen Hilfeleistung zum Gegenstand haben, so ist gegen diese These nichts einzuwenden. Wie bereits gezeigt, lassen sich auch normative Gegenstände optimieren. Doch diese reflexiven Gebote können zur Erläuterung oder Erklärung der nicht reflexiven Optimierungsgebote nichts beitragen. Es handelt sich schlicht um Gebote mit einem anderen Gegenstand. In unserer Rechtsordnung dürften sie eine Ausnahmeerscheinung sein. Entsprechend findet sich in Alexys Erläuterungen der Prinzipien auch kein konkretes Beispiel für entsprechend reflexive Gebote, geschweige denn eines aus einer konkreten Rechtsordnung. So sind auch die Grundrechte, die der Prinzipientheorie als Hauptanwendungsfall dienen, keine Normen, die gebieten, Normen zu optimieren, sondern - in der Lesart der Prinzipientheorie - Normen, die als Abwehrrechte gebieten, staatliche Zurückhaltung gegen- 
über Grundrechtsbeschränkungen zu optimieren, die zwar sowohl faktischer als auch normativer Art sein können, aber letztlich auf faktische Sachverhalte zielen.

Zudem macht die konstruktive Lesart der Möglichkeitsthese nur deutlich, dass es für den Optimierungscharakter eines Gebots gerade nicht auf den Gegenstand der Optimierung ankommt. Konstruktiv weist die Möglichkeitsthese nur darauf hin, dass neben faktischen Sachverhalten auch Normen optimiert werden können. Doch damit zeigte sie lediglich, dass Optimierungsgebote gerade nicht auf normative Gegenstände und schon gar nicht auf ein „ideales Sollen“ bezogen sein müssen. Zu ihrer Erklärung bedarf es daher auch keiner Prinzipien, die von Alexy mit dem „idealen Sollen" identifiziert werden.

\section{Notwendigkeitsthese}

Bereits Alexys These, dass sich Optimierungsgebote in der von ihm vorgeschlagenen Form mithilfe eines idealen Sollens rekonstruieren lassen, trifft nicht zu. Die von ihm vorgeschlagene Rekonstruktion bildet die Optimierungsgebote, die sie rekonstruieren soll, nicht ab, sondern führt zu Optimierungsgeboten, die nicht nur weitgehend praktisch irrelevant sind, sondern auch in keiner Weise erhellen können, in welchem Zusammenhang ein „ideales Sollen“ mit den Optimierungsgeboten stehen soll, um die es der Prinzipientheorie geht.

Doch auch wenn Alexys Vorstellung eines in Optimierungsgebote eingelassenen idealen Sollens auf normlogischen Irrtümern beruht und daher nichts zur Erklärung von Optimierungsgeboten beitragen kann, würde sich die Suche nach neuartigen normativen Entitäten lohnen, wenn Alexys zweite These zuträfe, dass wir Optimierungsgebote ohne die Annahme von ihnen verschiedener Prinzipien - was immer darunter zu verstehen wäre - nicht vollständig verstehen könnten. Die geradezu fieberhafte Suche der Prinzipientheoretiker nach neuen Prinzipienkonstruktionen jenseits der Optimierungsgebote hätte dann u.U. einen wissenschaftlichen Sinn. Wenn es etwas an Optimierungsgeboten gäbe, das wir nicht verstünden oder erklären könnten, so könnte dies damit zusammenhängen, dass wir zu ihrem Verständnis der Einsicht in besondere normative Entitäten bedürften, die Alexy mit dem idealen Sollen vielleicht noch nicht zutreffend erfasst hat, für die aber der der Regel gegenübergestellte Begriff des Prinzips gleichsam als Chiffre stünde.

Alexys Argument, warum Optimierungsgebote zur Erklärung etwa von als Optimierungsgebote interpretierten Grundrechten allein nicht ausreichen, geht dahin, „daß Grundrechte aus Normen bestehen und dass ihre Kollision deshalb adäquat nur als Normenkollisionen rekonstruiert werden kann. ... Man könnte meinen, dass das Normativitätsproblem auch dann lösbar sei, wenn man sich auf Optimierungsgebote beschränke. “41 Alexy hält diese Ansicht aber für falsch. Alexy meint, dass

41 Ebd. S. 30. 
Optimierungsgebote - für sich genommen - zwei Dinge nicht erklären könnten: zum einen die Normativität der Grundrechte im Rahmen der Optimierungsdogmatik der Prinzipientheorie und zum anderen das Kollisionsverhalten von Optimierungsgeboten.

\section{Das Normativitätsargument}

Zunächst sei gegenüber der Formulierung von Alexy die Klarstellung angebracht, dass Grundrechte nicht „aus Normen bestehen“, sondern Normen sind. Soweit bereits mit der Formulierung nahe gelegt werden soll, dass die Grundrechte einen normativen Gegenstand haben, da sie aus Normen „bestehen“, ist dies zurückzuweisen. Das grundrechtliche Abwehrrecht auf körperliche Unversehrtheit ist ein Unterlassungsgebot und als solches eine Norm; es hat keine Norm zum Gegenstand. Es hat das Unterlassen staatlicher Handlungen zum Gegenstand, die die Gesundheit beeinträchtigen. Grundrechtsbeeinträchtigungen sind keine Normen, sondern faktische Sachverhalte. Normen kommen als Grundrechtsbeeinträchtigung nur mittelbar in den Blick, soweit sie zu faktischen Grundrechtsbeeinträchtigungen ermächtigen. Doch der Gegenstand des grundrechtlichen Unterlassungsgebots ist letztlich kein normativer, sondern ein faktischer.

Dies beantwortet auch die Frage nach der Normativität der Grundrechte. Grundrechte sind normativ aufgrund des mit ihnen verbundenen Gebots, Grundrechtseingriffe zu unterlassen, nicht dadurch, dass sie sich auf ein weiteres Gebot beziehen. Werden Grundrechte als Optimierungsgebote verstanden, haben Sie - in der Notation Alexys - schlicht die Form:

OOpt $p$.

Dabei steht $p$ für das Unterlassen eines Grundrechtseingriffs, im Fall des Grundrechts auf körperliche Unversehrtheit etwa für das Unterlassen körperlicher Misshandlungen. Die Normativität des als Optimierungsgebot verstandenen Grundrechts wird durch den Gebotsoperator $O$ nicht nur ausreichend, sondern auch einzig zutreffend zum Ausdruck gebracht. Eine Verdopplung des Operators wäre nicht redundant, sondern würde den Grundrechten einen Gegenstand geben, der ihnen nicht entspricht. Art. 2 Abs. 2 GG verlangt nicht, das Gebot zu optimieren, Eingriffe in die körperliche Unversehrtheit zu unterlassen, sondern richtet sich unmittelbar auf das Unterlassen staatlicher Eingriffe in die körperliche Unversehrtheit. Zur Erklärung der Normativität von Optimierungsgeboten bedarf es keiner weiteren normativen Entitäten.

Aber auch Optimierungsgebote, die eine Norm zum Gegenstand haben, erhalten ihre Normativität allein aus dem normativen Operator, der sich auf die Optimierung bezieht, nicht aus dem normativen Operator des zu optimierenden Gebots. Dies zeigt sich, wenn der normative Operator durch einen deskriptiven ersetzt wird. Wird Es 
ist gesollt, dass ... durch Es ist eine Tatsache, dass ... ersetzt, wird der gesamte Ausdruck deskriptiv, auch wenn der Gegenstand normativ ist. Die Aussage, dass die Optimierung des Gebots von $p$ eine Tatsache ist, ist insgesamt deskriptiv. Auf die Normativität des Ausdrucks hat die Normativität des Gegenstands der Optimierung keinen Einfluss.

Die Normativität von Optimierungsgeboten gibt keinerlei Rätsel auf. Wie bei jeder Norm ergibt sie sich schlicht durch den normativen Operator, der sich auf die Optimierung eines Gegenstands - sei er faktisch oder normativ - bezieht.

\section{Das Kollisionsargument}

Auch die Erklärung von Kollisionen der Optimierungsgebote ist nicht auf eine Verdopplung ihrer Normativität angewiesen. Normkollisionen werden durch Kollisionstatbestände koordiniert. Diese Kollisionstatbestände lassen sich auch in die zu koordinierenden Normen einbauen, wie dies nach der Prinzipientheorie bei Optimierungsgeboten durch die Relativierung der Gebote auf die rechtlichen und faktischen Möglichkeiten anhand des Abwägungsgesetzes geschieht. Doch der Inhalt des Kollisionstatbestands ist kontingent und lässt die rechtslogische Struktur der Normen unberührt. Die Kollisionstatbestände sind in keiner Weise geheimnisvoll. Sie lassen sich wie jedes andere Tatbestandsmerkmal durch Auslegung und Subsumtion anwenden. Zu ihrem Verständnis bedarf es keines „idealen Sollens“. Dies lässt sich an zwei Normen demonstrieren, die Alexy als Beispiele für Prinzipien anführt. ${ }^{42}$ Die Norm N1 ermächtigt den Staat, zum Schutz seiner auswärtigen Belange Maßnahmen zu ergreifen. Die Norm N2 gewährt den Bürgern Pressefreiheit.

Wenn eine Handlung die auswärtigen Belange des Staates gefährdet, dann soll sie verboten werden.

Wenn eine Handlung der Presse dient, dann soll der Staat Verbote unterlassen.

Für beide Normen gibt es eine Schnittmenge von Sachverhalten, in denen sie beide anwendbar sind. Für diese Schnittmenge von Sachverhalten muss die Rechtsordnung einen Kollisionstatbestand einführen, der in den Tatbestand der kollidierenden Normen eingearbeitet werden kann. Welchen Inhalt der Kollisionstatbestand hat, ist gänzlich kontingent. Ein traditioneller Kollisionstatbestand ist etwa der Lex-posterior-Grundsatz $\left(\mathrm{K}_{\mathrm{a}}\right)$. Entstammte etwa die Norm N1 über den Staatsschutz noch der Zeit einer Militärdiktatur und die Norm N2 der Zeit nach dem demokratischen Umbruch, so ist der Lex-posterior-Grundsatz selbst für die Normen N1 und N2 nicht ausgeschlossen. Dies würde dazu führen, dass - falls N1 nicht mit der neuen Ordnung gänzlich unvereinbar wäre - N1 interpretatorisch um einen Ausnahmetatbestand ergänzt würde. 
Wenn eine Handlung die auswärtigen Belange des Staates gefährdet und diese Handlung nicht der Presse dient, dann soll sie verboten werden.

Wenn eine Handlung der Presse dient, dann soll der Staat Verbote unterlassen.

Durch das Einfügen des Kollisionstatbestands bleibt die normative Struktur der kollidierenden Normen unverändert. Der Kollisionstatbestand führt besonders nicht dazu, dass die Normen einen reflexiven Charakter in dem Sinn erhalten, dass sie nicht nur Gebote sind, sondern sich auch noch auf ein Gebot als Gegenstand beziehen. Sie haben weiterhin die Struktur

$\mathrm{Op}$

nicht

O Op.

Daran ändert sich auch nichts, wenn der Kollisionstatbestand Elemente des Gewichts aufweist. Etwa könnte eine teleologische Reduktion von N1 ergeben, dass sie nur für erhebliche auswärtige Belange gelten soll. Nach diesem Kollisionstatbestand $\mathrm{K}_{\mathrm{b}}$ ergäbe sich:

Wenn eine Handlung erhebliche auswärtige Belange des Staates gefährdet, dann soll sie verboten werden.

Wenn eine Handlung der Presse dient und erbebliche auswärtige Belange des

Staates nicht gefährdet, dann soll der Staat Verbote unterlassen.

Wie bei Optimierungsgeboten bedarf es bei $\mathrm{N} 1_{\mathrm{b}}$ und $\mathrm{N} 2_{\mathrm{b}}$ der Gewichtung von Belangen. Im Unterschied zu Optimierungsgeboten ist die Gewichtung jedoch nicht relativ zu einem andern Belang. Es muss lediglich festgestellt werden, ob der gefährdete auswärtige Belang ein erheblicher ist. Auch bei diesem Kollisionstatbestand verändert sich die Struktur der Normen nicht. Es tritt auch kein Phänomen auf, das nur mit Hilfe eines „idealen Sollens“ oder ohne ein sonst mit Prinzipien im Sinn der Prinzipientheorie in Verbindung gebrachtes Phänomen wie „unendlich iterierten Geltungsgeboten" verständlich gemacht werden könnte.

Von dem Kollisionstatbestand $\mathrm{K}_{\mathrm{b}}$ ist es nur noch ein kleiner Schritt zu einem Kollisionstatbestand, wie ihn Optimierungsgebote enthalten. Wird in einer Kollisionsregel $K_{b}$ statt auf „erhebliche“ auf „überwiegende“ auswärtige Belange abgestellt, entstehen zwei Normen, die eine Abwägung zwischen der Gefährdung der auswärtigen Belange und der Beeinträchtigung der Pressefreiheit fordern, wie sie das Abwägungsgesetz verlangt, mit dem die Prinzipientheorie Optimierungsgebote operationalisiert. $^{43}$ 
Wenn eine Handlung überwiegende auswärtige Belange des Staates gefährdet, dann soll sie verboten werden.

Wenn eine Handlung der Presse dient und überwiegende auswärtige Belange des Staates nicht gefährdet, dann soll der Staat Verbote unterlassen.

Die semantische Verschiebung von „erheblich“ zu „überwiegend“ lässt die Struktur der Normen aber ebenso unangetastet wie die anderen Kollisionstatbestände.

Ihr Gegenstand ist weiterhin ein faktischer, kein normativer. Auch wenn der Kollisionstatbestand eine relative Gewichtung in Form einer Optimierung verlangt, zeigen die Normen keine Eigenschaften, zu deren Erklärung die Annahme eines idealen Sollens oder sonstiger zusätzlicher normativer Entitäten etwas beitragen müsste oder auch nur könnte. Weder haben Optimierungsgebote die ihnen von Alexy unterstellte Struktur, noch sind Optimierungsgebote gegenüber anderen Normen mit Kollisionstatbeständen durch besondere normstrukturelle Eigenschaften ausgestattet, die die Suche nach besonderen normativen Entitäten erforderlich machte. Für eine Theorie der Grundrechte etwa, die alle Grundrechtskonflikte durch optimierende Abwägung zu lösen vorschlägt, reichte eine Rekonstruktion der Grundrechte als Optimierungsgebote vollständig aus. Der Annahme von Prinzipien, die nicht mit den Optimierungsgeboten identisch sind, bedarf es nicht.

\section{Der Existenzeinwand}

Worüber reden Prinzipientheoretiker nun, wenn sie von Prinzipien sprechen? Was ist mit der mysteriös klingenden Rede vom „idealen Sollen“ gemeint? Was Alexy im Rahmen der Prinzipientheorie als „ideales Sollen“ zu rekonstruieren versucht, sind unbedingt formulierte Normen wie N1 und N2, die nicht explizit durch Kollisionstatbestände auf rechtliche und faktische Rahmenbedingungen relativiert sind. Das Gebot, Notleidenden zu helfen, kann für ihn auch als Regel im Sinn der Prinzipientheorie verstanden werden, doch hätte es dann zur Konsequenz, dass den Notleidenden in jedem Fall geholfen werden müsste ${ }^{44}$ - etwa auch unabhängig von den Kosten und Risiken der Hilfe für die Helfer. Doch eine entsprechende wörtliche Interpretation scheint den Vertretern der Prinzipientheorie zu Recht häufig unplausibel. Um den unbedingten Normformulierungen dennoch einen Sinn zu verleihen, werden sie als „,ideales Sollen“, als unbedingtes Sollen im Bereich des Idealen, interpretiert. In der Idealität können sie ihrem unbedingten Wortlaut gerecht werden, weil sie nicht auf die faktischen und rechtlichen Möglichkeiten der Realität relativiert werden müssen. Im Bereich des realen Sollens sollen die Prinzipien dann die Form von Optimierungsgeboten erhalten. 
So anerkennenswert das Bemühen der Prinzipientheorie ist, den Wortlaut von Normformulierungen ernst zu nehmen, handelt es sich bei der prinzipientheoretischen Dialektik von idealem und realem Sollen doch nur um eine unnötig umwegige Interpretation einer Normformulierung, die zudem noch die Interpretationsmöglichkeiten unzulässig verengt. Normformulierungen wie N1 und N2 oder das unbedingt formulierte Hilfeleistungsgebot sind verkürzte oder unvollständige Ausdrücke für komplexere Tatbestände, die durch Interpretation zu praktikablen Normen entwickelt werden müssen, wobei der historische Kontext der Normen, ihre Genese, ihr systematischer Zusammenhang, dogmatische Traditionen etc. die maßgeblichen Parameter bilden. ${ }^{45}$ Aus der diesen allgemeinen methodischen Standards folgenden Interpretation der jeweiligen Normformulierungen ergeben sich dann auch Kollisionstatbestände, die die Normen in ein Verhältnis zu anderen Normen desselben Normsystems setzen.

Anders als die umwegige Interpretation der unbedingten Normformulierungen als „,ideales Sollen“, das alle Prinzipien auf Optimierungsgebot reduziert, dies glauben machen will, können die Kollisionstatbestände, die sich aus einer klassischen Interpretation unbedingter Normformulierungen ergeben, die unterschiedlichsten Formen annehmen und sind - wie das kleine Beispiel zu N1 und N2 zeigt - nicht auf einen bestimmten Kollisionstatbestand festgelegt.

Selbst dort, wo der Kollisionstatbestand auf eine Verhältnismäßigkeitserwägung hinausläuft, ist damit noch nicht bestimmt, welche Interpretation des Verhältnismäßigkeitsgrundsatzes zugrunde gelegt werden soll, auch wenn die Prinzipientheorie meint, ihn für sich einnehmen zu können: „Die Prinzipientheorie impliziert den Verhältnismäßigkeitsgrundsatz und dieser jene." 46 Doch auch der Verhältnismäßigkeitsgrundsatz muss nicht als Optimierungsgebot verstanden werden. Jenseits des pareto-optimalen Erforderlichkeitskriteriums kann er auch als Garantie einer Mindestposition $^{47}$ oder als Verbot grober Disproportionalität ${ }^{48}$ verstanden werden. Von einer ganzen Reihe von Autoren werden Verfassungsprinzipien allgemein nicht als Optimierungsgebote, sondern als Mindest- oder Rahmengarantien interpretiert. ${ }^{49}$ Wie die Interpretation der elliptischen Normformulierungen ausfällt, ist keine Frage, für die die von der Prinzipientheorie postulierte Unterscheidung von Regeln und Prinzipen etwas austragen kann. Welcher Kollisionstatbestand etwa N1 und N2 ko-

45 Vgl. Rusteberg, Gewährleistungsgehalt (Fn. 9), S. 158-166, der zeigt, wie die klassischen Auslegungsgesichtspunkte bei der Bestimmung des Schutzbereichs der Grundrechte von der Prinzipientheorie überspielt werden.

46 Alexy, Struktur (Fn. 16), S. 31 (35).

47 B. Schlink, Abwägung im Verfassungsrecht, Berlin 1976, S. 76-78, 192-195.

48 B. Pieroth/B. Schlink, Grundrechte Staatsrecht II, 26. Aufl. Heidelberg 2010, Rn. 304; Poscher, Abwehrrechte (Fn. 9), S. 224.

49 K.-E. Hain, Die Grundsätze des Grundgesetzes, Baden-Baden 1999, S.157; F. Reimer, Verfassungsprinzipien, Berlin 2001, S. 329-333, 338-348 jeweils m.w.N. 
ordiniert, ist kontingent. Es ist sogar kontingent, ob eine unbedingt formulierte Norm überhaupt durch einen Kollisionstatbestand eingeschränkt wird. Für Art. 1 Abs. 1 GG war die negative Antwort etwa lange Zeit weitgehend unumstritten. ${ }^{50}$

Das „ideale Sollen“ Alexys hat nicht Normen, sondern verkürzte oder unvollständige Formulierungen von Normen zum Gegenstand, die jedoch nicht zu einem „idealen Sollen “ stilisiert, sondern lediglich interpretiert werden müssen. So wie der deskriptive Satz „Kunst und Wissenschaft ... sind frei.“ als verkürzte Formulierung für ein Grundrecht steht, die überhaupt erst als Norm, dann als Abwehrrecht gegen staatliche Eingriffe und schließlich als auf der Grundlage kollidierenden Verfassungsrechts beschränkbar interpretiert werden muss. Doch ebenso wenig wie es zur Interpretation des deskriptiv formulierten Art. 5 Abs. 3 S. 1 GG der Annahme einer besonderen Art „faktischen Sollens“ bedarf, bedarf es für die Interpretation unbedingt formulierter Normen der Annahme eines „idealen Sollens“. In beiden Fällen bedarf es lediglich der Auslegung der konkreten Normformulierung in ihrem Kontext. Letztlich hypostasiert die Prinzipientheorie verkürzte Normformulierungen zu einer normativen Entität.

\section{Warum Optimierungsgebote der Prinzipientheorie nicht ausreichen}

Der einzige Gegenstand, mit dem sich Prinzipien im Sinn der Prinzipientheorie sinnvoll identifizieren lassen und die ursprünglich auch von Alexy mit ihnen identifiziert wurden, sind Optimierungsgebote. In allen praktischen Anwendungsfällen der Prinzipientheorie geht es um die Interpretation von Rechtsnormen als Optimierungsgebote und das zur Optimierung herangezogene Abwägungsgesetz. Warum kann sich die Prinzipientheorie also nicht damit begnügen eine Theorie der Optimierungsgebote zu sein? Warum kann sie nicht wie ehedem Prinzipien mit Optimierungsgeboten identifizieren und sich der bemühten weiteren Versuche zur Rekonstruktion von Prinzipien entschlagen, deren Wert auch Prinzipientheoretikern zunehmend fraglich erscheint $^{51}$ ? Wieso kann die Prinzipientheorie nicht schlicht eine Theorie der Optimierungsgebote sein?

Die Antwort liegt in der strategischen Funktion, die der von der Prinzipientheorie postulierte Dualismus von Regeln und Prinzipien in dem Projekt der Prinzipientheorie einnimmt. ${ }^{52}$ Wenn sich alle Normen rechtstheoretisch in unmittelbar anwendbare Regeln auf der einen und Prinzipien auf der anderen Seite einteilen lassen, Prinzipien

50 S. R. Poscher, Die Würde des Menschen ist unantastbar, Juristenzeitung 2004, S. 756-762 m.w.N.

51 So resümiert etwa Borowski, Prinzipien (Fn. 1), S. 105, seine Darstellung der unterschiedlichen Vorschläge zum Prinzipienbegriff: "Bei aller weiteren, aus rechtstheoretischer Sicht zu begrüßenden Präzisierung der Prinzipientheorie darf jedoch nicht übersehen werden, daß mit steigender Kompliziertheit ein entscheidender Vorteil der Prinzipientheorie, ihre intuitive Plausibilität, zunehmend in den Hintergrund tritt.“

52 Dazu auch K. Möller, Balancing and the Structure of Constitutional Rights, International Journal of Constitutional Law 5 (2007), S. 453 (457f.). 
als Optimierungsgebote zu verstehen und im Wege der Abwägung anzuwenden sind, dann geht mit der Qualifikation einer Norm als Prinzip immer auch schon eine bestimmte Anwendungsdogmatik, nämlich die der Abwägung einher. Wenn der Regelbegriff auf der anderen Seite so definiert wird, dass er nur dann einschlägig ist, wenn sich keine Anwendungsprobleme stellen, sondern es nur noch der bloßen Subsumtion bedarf, dann können alle ernsthaften Rechtsanwendungsfragen zu Abwägungsfragen erklärt werden. Normen, die nicht durch einfache Subsumtion anwendbar sind, sind Prinzipien oder haben jedenfalls ein Prinzipienelement; sie sind daher im Wege der Abwägung anzuwenden. Durch den postulierten normativen Dualismus sollen umstrittene Auslegungsfragen rechtstheoretisch zugunsten einer Abwägungsdogmatik entschieden werden. Art. 1 Abs. 1 GG ist keine unmittelbar subsumptionsfähige Regel, es muss sich daher in der Strategie der Prinzipientheorie um ein Prinzip handeln. Prinzipien implizieren jedoch Optimierungsgebote. Art. 1 Abs. 1 GG steht daher grundsätzlich auch unter dem Abwägungsgesetz und daher der Abwägung offen. ${ }^{53}$ Positionen, die etwa von der Abwägungsfestigkeit der Menschenwürdegarantie ausgehen, können daher als (prinzipien-)theoretisch widerlegt gelten.

Wenn Prinzipien hingegen lediglich Optimierungsgebote sind, dann sind Prinzipien jedoch auch nach der Prinzipientheorie lediglich Regeln. Wenn aber Prinzipien Regeln sind, bricht der normative Dualismus, auf dem die Prinzipientheorie beruht, in sich zusammen. Dann gibt es nur noch Normen, die je nach Interpretation einen unterschiedlichen Inhalt haben können. Dieser Inhalt kann nicht mehr theoretisch anhand eines Prinzipiencharakters bestimmt werden. Damit lässt sich dann auch nicht mehr theoretisch bestimmen, ob die Menschenwürdegarantie der Abwägung zugänglich ist oder als absolutes Verbot verstanden werden muss. Dies heißt nicht, dass sich nicht auch weiterhin etwa für eine Optimierungsdogmatik der Grundrechte argumentieren ließe, nur müsste sich die Argumentation dann der herkömmlichen juristischen Methoden bedienen. Die Dogmatik der Grundrechte ließe sich nicht mehr anhand ihres Prinzipiencharakters auf eine optimierende Abwägung festlegen, sondern es müsste dogmatisch dafür argumentiert werden, dass eine Interpretation der Grundrechte als Optimierungsgebote trotz der dagegen vorgebrachten dogmatischen Bedenken gegenüber anderen Vorschlägen vorzugswürdig ist.

Es geht hier nicht darum, welche Interpretation der Grundrechte vorzugswürdig ist. Es geht nur darum, zu zeigen, welche strategische Funktion der normative Dualismus der Prinzipientheorie hat. Wenn die Prinzipientheorie sich damit begnügen würde eine Theorie der Optimierungsgebote zu sein, müsste sie den von ihr postulierten normativen Dualismus und damit aber auch ihre theoretisch ansetzende, aber letztlich auf konkrete Dogmatik zielende Argumentationsstrategie aufgeben. Sie wäre

53 Zur prinzipientheoretischen Interpretation der Menschenwürdegarantie N. Teifke, Flexibilität der Menschenwürde?, ARSP Beiheft Nr. 103 (2005), S. 142 ff. 
dann nur noch eine dogmatische Theorie unter vielen, gegen die jedenfalls im Bereich der Grundrechtsdogmatik fast alle Kriterien sprechen, die - u.a. von Alexy selbst in seinen früheren Arbeiten ${ }^{54}$ - für die Qualität dogmatischer Theorien entwickelt wurden. ${ }^{55}$ Argumentationsstrategisch hängt für die Prinzipientheorie an dem Postulat des normtheoretischen Unterschieds zwischen Optimierungsgeboten und Prinzipien alles. Dies mag erklären, warum sich die Prinzipientheoretiker in für Außenstehende kaum noch nachvollziehbarer Weise an Vorschlägen überbieten, die Illusion eines Unterschieds zwischen Prinzipien und Optimierungsgeboten zu stabilisieren - trotz aller besonders auch theorieinternen Kritik an den jeweiligen Ansätzen.

\section{E. Der gute Sinn der Rede von Rechtsprinzipien}

Es gibt keine Prinzipien im Sinn der Prinzipientheorie, die sich von Optimierungsgeboten unterscheiden und die in irgendeiner Weise Relevanz für die Erklärung von Optimierungsgeboten entfalten würden. Gemessen an ihren eigenen Kriterien ist die Prinzipientheorie eine Theorie ohne Gegenstand, die Theorie eines Phantoms. Dies bedeutet indes nicht, dass die Rede von Rechtsprinzipien im traditionellen Sinn keinen Gegenstand hätte, dass es auch keine Prinzipien im traditionellen Sinn gäbe. Im Gegenteil, es gibt weitaus mehr Prinzipien als die Prinzipientheorie mit ihren auf Optimierungsgebote verengten Prinzipienbegriff zulassen will. ${ }^{56}$ Es trifft zwar zu, dass sich einige Rechtsgrundsätze - etwa der Grundsatz des Vertrauensschutzes unter Umständen auch als Optimierungsgebot rekonstruieren lassen. Doch gilt dies längst nicht für alle Rechtsgrundsätze. Der Grundsatz von „Treu und Glauben“ verlangt nicht Optimierung, sondern schlicht Beachtung: Wenn etwas gegen Treu und Glauben verstößt, dann kommt es auf eine Abwägung nicht mehr an. ${ }^{57}$ Auch der Gleichheitsgrundsatz mit seiner phänomenologisch komparativen Perspektive lässt sich nicht in das Korsett eines Optimierungsgebots zwingen, ohne dass gerade dieses spezifische Element verloren ginge. ${ }^{58}$ Nicht zuletzt zeigt sich die Diversität der Rechtsprinzipien auch am Grundsatz der Verhältnismäßigkeit, für den neben der von der Prinzipientheorie vorgeschlagenen Interpretation im Sinn eines Optimierungsgebots, jedenfalls noch das Verständnis als Verbot grober Disproportionalität und der Mindestgarantie im Raum stehen, wobei sich die unterschiedlichen Interpretationen

54 R. Alexy, Theorie der juristischen Argumentation, 3. Aufl. Frankfurt 1996, S. 326.

55 Dazu eingehend Poscher, Abwehrrechte (Fn. 9), S. 81 f. u. 10-13.

56 Dies gilt auch für den Bedeutungs- und Facettenreichtum, der dem Begriff „Prinzip“ in der philosophischen Tradition zukommt, die bei dem griechischen „Arché" einen ihrer begrifflichen Ausgangspunkte hat, zur eindrucksvollen Begriffsgeschichte Reimer, Verfassungsprinzipien (Fn. 49), S. 146-71, der schon aus begriffsgeschichtlichen Gründen eine Verkürzung des Prinzipienbegriffs auf „Abwägungsfähigkeit“ ablehnt, ebd. S. 179.

$57 \mathrm{Zu}$ diesem Einwand mit weiteren Beispielen auch O. Weinberger, Revision des traditionellen Rechtssatzkonzepts, in: B. Schilcher/P. Koller/B.-C. Funk (Hrsg.), Regeln, Prinzipien und Elemente im System des Rechts, Wien: Verlag Österreich 2000, S. 53 (64).

58 Vgl. dazu A. Somek, Eine egalitäre Alternative zur Güterabwägung, in: B. Schilcher/P. Koller/B.-C. Funk (Hrsg.), Regeln, Prinzipien und Elemente im System des Rechts, 2000, S. 193-220. 
nicht grundsätzlich ausschließen, sondern in unterschiedlichen Kontexten unterschiedlich zu bewerten sein könnten.

Die Welt der Prinzipien ist viel reicher und bunter, als die Prinzipientheorie es mit ihrer Verengung des Prinzipienbegriffs auf Optimierungsgebote postuliert. Anders als die Prinzipientheorie es glauben machen will, ist der Unterschied zwischen Prinzipien und anderen Normen auch kein struktureller, sondern ein gradueller. ${ }^{59}$ Als Rechtsprinzipien lassen sich Normen bezeichnen, die neben einer besonderen Konkretisierungsbedürftigkeit eine besondere meist auch rechtsgebietsübergreifende Bedeutung entfalten oder die, wie Josef Esser es für den Bereicherungsgrundsatz gezeigt hat, die Ausdifferenzierung einer ganzen Dogmatik leiten können. ${ }^{60}$ Nichts spricht dagegen, Normen, die diese Merkmale in besonders ausgeprägter Weise aufweisen, mit dem Begriff des Rechtsprinzips hervorzuheben. Doch bedeutet dies nicht, dass alle anderen Normen sich immer durch schlichte Subsumtion anwenden ließen und nur Rechtsprinzipien konkretisierungsbedürftig wären. Für fast alle Normen lassen sich Anwendungsfälle denken, in denen sie der Konkretisierung bedürfen, und viele Normen verwenden abstrakte Begriffe, die in vielen Fällen nicht durch schlichte Subsumtion anwendbar sind. Der Unterschied zwischen Rechtsprinzipien und sonstigen Rechtnormen ist daher graduell in den Dimensionen der Konkretisierungsbedürftigkeit und der Bedeutung einer Norm für die Rechtsordnung.

Der neuerliche Versuch Alexys, den normativen Dualismus der Prinzipientheorie durch Modifikationen und Erläuterungen des ihr eigenen Prinzipienbegriffs zu stützen, überzeugt nicht. Die nun vorgebrachten Überlegungen lassen nur noch einmal deutlich werden, wie verfehlt das Insistieren auf nicht mit Optimierungsgeboten identischen Prinzipien als einer eigenen normativen Entität im Bereich eines“idealen Sollens “ ist. Die Argumentation verstrickt sich in deutlich hervortretende Fehler oder bestenfalls in Trivialitäten, die zur Erklärung der die Prinzipientheorie beschäftigenden normativen Phänomene nichts beitragen. Statt sich in idiosynkratischen Phantomdebatten zu verlieren, die um einen Gegenstand kreisen, den es nicht gibt, sollten

59 Jenseits des Kreises exponierter Vertreter der Prinzipientheorie wird dies auch fast einhellig so gesehen, etwa P. Lerche, Übermaß und Verfassungsrecht, 2. Aufl. Köln, Berlin, Bonn, München 1999, S. XXII: „Unterscheidung dieser beiden Kategorien m.E. nur quantitativer Natur“; B. Schilcher, Prinzipien und Regeln als Elemente einer Theorie des gebundenen Ermessens, in: B. Schilcher/P. Koller/B.-C. Funk (Hrsg.), Regeln, Prinzipien und Elemente im System des Rechts, 2000, S. 153 (164, 169); Reimer, Verfassungsprinzipien (Fn. 49), S. 179-182; in der angelsächsischen Literatur so bereits H. L. A. Hart, The Concept of Law, (1961), 2. Aufl. Oxford: Clarendon Press 1994, S. 259 f.; A. Marmour, The Separation Thesis and the Limits of Interpretation, The Canadian Journal of Law and Jurisprudence 12 (1999), S. 135 (145 f.); M. S. Moore, Legal Principle Revisited, in: M. S. Moore, Educating Oneself in Public, New York: Oxford University Press 2000, S. 221 (225); M. Bódig, Rules, Principles, and the Problem of the Limits if Legal Reasoning, 2008, abrufbar unter SSRN: http://ssrn.com/abstract $=1318638$, S. 4; zu einer möglichen rechtslogischen Rekonstruktion des graduellen Unterschieds B. Verheij/J. Hage/H. J. van den Herik, An integrated view on rules and principles, Artificial Intelligence and Law 6 (1998), S. 3-26; eine dekonstruktive Lesart der Unterscheidung findet sich bei $P$. Schlag Rules and Standards, UCLA Law Review 33 (1985), S. 379 (405-30).

60 J. Esser, Grundsatz und Norm in der richterlichen Form des Privatrechts, 4. Aufl., 1990, S. 154. 
die Anhänger der Prinzipientheorie ihre Theorie als das verstehen - und ehrlicher Weise wohl auch bezeichnen -, was sie ist: eine Theorie der Optimierungsgebote. Soweit sie sich dogmatischen Gegenständen zuwenden, ${ }^{61}$ handelt es sich jeweils um Vorschläge einer Optimierungsdogmatik, die auf unterschiedlichen Feldern wie etwa dem Planungsrecht oder den Grundrechten ganz unterschiedlich zu bewerten sind. Als Beitrag zu unterschiedlichen dogmatischen Debatten lässt sich für eine Optimierungsdogmatik jedoch nicht von der vermeintlich hohen Warte der Rechtstheorie aus argumentieren. Optimierungsdogmatiker müssten vielmehr in dem jeweiligen Feld mit dogmatischen Argumenten überzeugen.

$61 \mathrm{Zu}$ anderen etwa argumentationstheoretischen Gegenständen der Prinzipientheorie Poscher, Einsichten (Fn. 5), S. 73-7; ders. Insights (Fn. 5), S. 441-45; ders. Principles (Fn. 5), S. 33-9. 